Products and Services In Practice is provided to readers using text and images from the manufacturer, supplier or distributor and does not imply endorsement by $B D J$ In Practice. Normal and prudent research should be exercised before purchase or use of any product mentioned.

Please send product and services news through to David Westgarth, BDJ In Practice via: David.Westgarth@bda.org

\section{Power harnessed}

High performance ceramics like zirconia have become hugely popular as crown and bridge materials thanks to their unique properties, but can be difficult to work on with conventional instruments.

DIATECH Z-Rex diamond instruments have been designed and manufactured by COLTENE to remedy this. Sharp and resilient, the innovative diamond instruments make it easy to adjust, trepan and remove ceramic restorations and shorten procedure times for zirconia crown and bridge removal.

In addition to a synthetic diamond mixture, all instruments have revolutionary Enhanced Resilient Alloy (ERA) bonding that has exceptional properties helping to reduce the delamination of diamonds from the shank for higher durability and efficiency.

To find out more about DIATECH Z-Rex diamond instruments available in both medium and coarse grits, and for expert advice, visit www.coltene.com, emailinfo.uk@coltene.com or call 01444 235486.

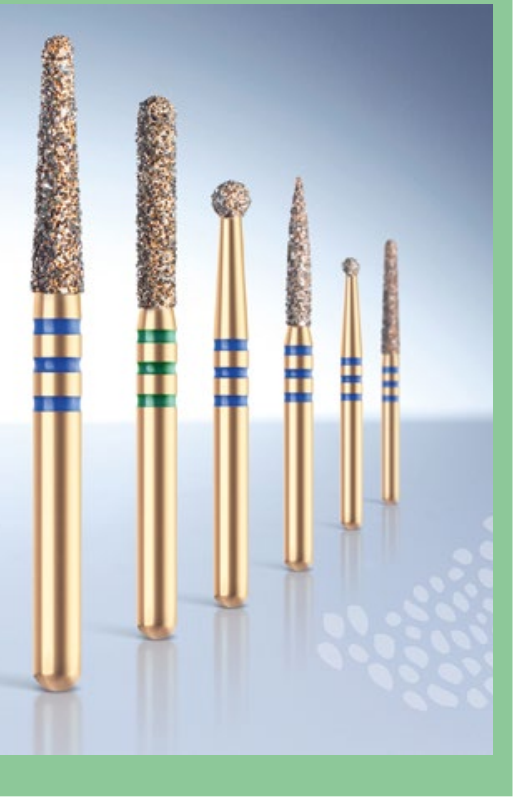

\title{
All inclusive
}

Behind every good business are good people. It is important for all members of the team to broaden their skills and scope of practice by attending industry events such as this year's BDIA Dental Showcase (17-19 October 2019, Birmingham NEC). This all-inclusive event offers something for everyone.

Firstly, there are over 300 exhibitors attending, who will be showcasing everything from sundries to digital equipment. They'll be plenty to peruse, and every possible individual interest and specialty will be catered for. Rather than try and grab five minutes with a rep between appointments, you'll be able to really grill the trade on the latest innovation and look, touch, feel, and 'play' with the products. Dental Showcase has more trade exhibitors than any other industry event. What's more, it takes place over three days - Thursday, Friday and Saturday - so you can schedule the team's attendance, meaning no loss of business.

New this year, for the 'techies', is a Digital Workflow Theatre, sponsored by DD, where you'll be able to have a peak into 'tomorrow's world' and see how technology is changing within dentistry. There will be

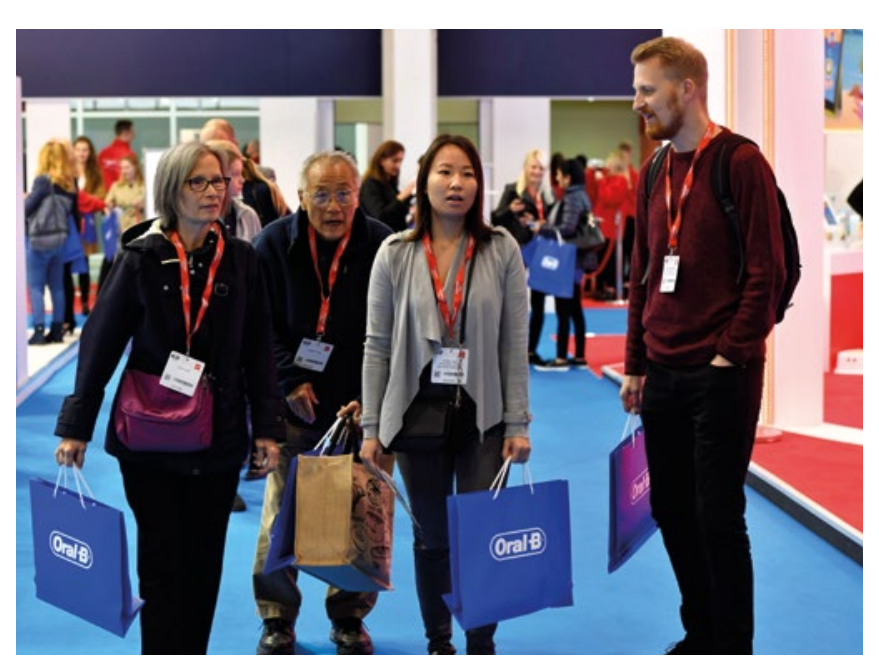

plenty of hands-on opportunities, ideal if you're considering any capital purchases or simply want to add a few items to your 'wish list'.

Also new for 2019 is the Practice Owners' Lounge, sponsored by Dentsply Sirona. Complimentary refreshments will be served throughout the day in this exclusive environment, giving principals an ideal area to have informal talks with peers or professionals in the dental trade in a relaxed, private environment.

Something all team members need to be aware of are the GDC standards and how to ensure compliance; nobody wants to fall foul of these regulations and ignorance is never an acceptable defence. The safeguarding of vulnerable adults is another example of a lecture relevant to all practices. Identifying concerns and knowing what protocols to follow will empower the whole dental team.

You can top up your CPD with practical lectures giving insight into the latest research on procedures you employ daily. This is the remit for this year's Dental Update Theatre, sponsored by DMG, where the focus will be on restorative topics, providing visitors with relevant knowledge they can apply in practice.

\section{All industry} associations will be represented under one roof in a designated 'Associations Hub'. If you've ever wanted to have your say with the BDIA, this is the place to do it.

To register visit dentalshowcase. com/register. 